\title{
Virtual Machine Consolidation Method with Low Delay and Energy Consumption Parameters
}

\author{
Nayana D. \\ M.Tech-Software Engineering \\ Department of Information Science and Engineering \\ M S Ramaiha Institute of Technology
}

\author{
Mydhili K. Nair, PhD \\ PROFESSOR and UG Programme Coordinator \\ Department of Information Science and Engineering \\ M S Ramaiha Institute of Technology
}

\begin{abstract}
There is huge amount of information available and maintaining such a data will require lot of computational power and the solution for these issues is to provide a system with more flexible, reliable and scalable characters. Cloud based orchestration services support the users any time and also can be scaled based on the demand for resources. Demand and delay are directly proportional to each other and if the duration of the delay is exponential then the cost also is also exponential. Performing a balance act between the quality of service and energy consumption rate is proposed by the algorithm. Each of the data center used in the cloud have set of virtual machines. In order have better processing of data consolidation of virtual machines is done. The proposed method is built on a fuzzy logic with consolidation framework. The method filters the virtual machine from an overloaded host and the migration control mechanism build into the system helps in increasing the performance for the selection process. Three characteristics namely mean, standard deviation and media are taken to compute the overload ratio due the selection process to improve optimization.
\end{abstract}

\section{Keywords}

Virtual Machine Consolidation

\section{INTRODUCTION}

The pay-as-you-go services provide a new domain for the IT companies to have dynamic provision for the computational services with the help of virtual systems. The environments which are in isolation are consolidated and provide a pool of opportunities for the cloud business. There is a shifting gradient between dedicated hardware to a shared cloud. Data warehousing technology is used to meet demand which in turn requires huge amount of energy. The initial stages for cloud concentrated on providing the computing needs and hence lead to energy consumption. By adopting virtual machine consolidation has taken more energy could be conserved by shutdown underutilized datacenters. Two ways are present in order to achieve the goal. The first way is to perform the migration from VM 'A' to $\mathrm{VM}$ 'B' and the second way is to consolidate the VMs The consolidation of virtual machines is the main area of research. Less amount of energy consumption happens for virtual machine whose status is inactive or if the virtual machines are in sleep mode. For duration of $\mathrm{T}$ the energy consumption can be reduced. If the data centers utilization ratio is less then such data warehouses can be shut down so that more energy is utilized on highly used virtual machines. A better approach would be to perform consolidation of virtual machine of one server and then perform the migration to a virtual machine of other location. Multiple kinds of cloud environment exists which can be private, public or hybrid and cloud provides different kind of models. The amount of electric current used during the year 2012 is 300-400TWh and will increase by triple times for the year 2020 [1, 2] as shown in Fig.1.[3] The amount of energy used for cooling and switching on the IT equipment is around $88 \%$. A work on the energy reduction process can provide both cost as well as power savings. As per the Amazon Web Services statements the energy cost for data centers is $42 \%$ of total operational cost [4].

There are also environment concerns when the energy consumption is more then $\mathrm{CO} 2$ release is more and hence causes harm to environment [5].Data Centers have a large set of $\{\mathrm{PM} 1, \mathrm{PM} 2, \ldots . ., \mathrm{PMn}\}$. Where each PMi, represents the Physical Machine. The PMi is future distributed across multiple management groups. The group can either be homogenous or it can be heterogeneous [5]. The combination of virtual resources along with an operating system and application programs set form a virtualized environment known as Virtual Machines (VMs). The VMs are internal liked to different PMs and are working on task with Service Level Agreement (SLA) provided by clients. In order to reduce the power consumption and make efficient use of resources based on demand metrics multiple virtual servers can be used $[6,7]$.

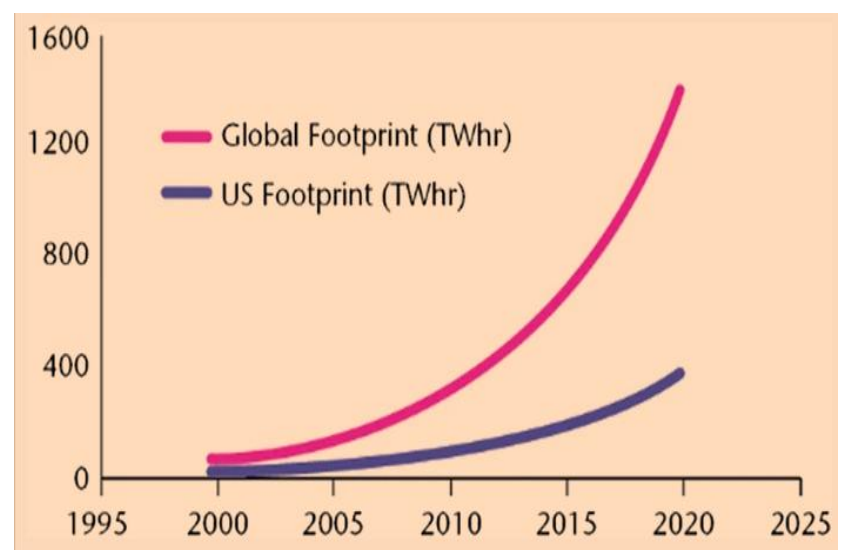

Fig1. Energy Consumption Projection Curve

The rest of the paper is organized as follows. Section 2 provides high level overview of the research or approaches which are present in the literature to reduce energy consumption on cloud. Section 3 describes the proposed method. Section 4 describes the results obtained from the implementation and final section provides the conclusion.

\section{BACKGROUND}

There are multiple strategies which are available for performing the energy consumption reduction and efficiency improvement namely Resizing of VMs, Placement Approach, Physical Resource Handles and Consolidation of VMs. 
Resizing of VMs involves addition and subtraction of resources or capacity fluctuation based on demand. The entire process happens in such a way that the VMs need not. Perform reboot or reconfigured or recreated [8]. The load is studied and PMi ( $\mathrm{i}=1$ to $\mathrm{N}$ ) are tuned to reduce the power consumption $[9,10]$

The resource elements can be added or removed, the capacity of the resource can be adjusted during VM Resizing. The VM need not be restarted or reconfigured. The load adjustment is done by the PMs and also reduces the amount of power consumption $[11,12]$.

In Placement Approach the individual VM or a group of VMs are assigned to servers during the initialization process in such a way that traffic load is managed to reduce energy or to lessen then total number of inter rack PMis. The placement issues can be solved by making use of approaches like Ant Colony system based approach, Ant Colony Optimization (ACO) based Virtual Machine (VM) placement approach etc $[13,14]$

The PMs are taken out and then VMs are created on top of PMs with a value of ratio higher than 1:1. This approach makes use of idle resources present in the PM. The quality of Service must also be maintained at acceptable levels for better utilization of PM to cater for Quality of Service (QoS) requirement [15].

The optimization function which reduces number of active PMs by migrating VMs over a period of time in an optimized fashion which in turn leads to reduce consumption of resources is called as VM consolidation [16]. Apache CloudStack is a platform used by many users and it also contains a suite of software. The set of VMs are created to move them to appropriate PMs so that it can run in an efficient manner. The framework of VM consolidation makes use of package creation algorithms, monitoring the resources and also performing the balanced approach for utilization of CPUs [17]. The K-means method helps in providing VM consolidation and is very helpful for a dynamic environment [18]. Monitoring is one of the important tasks in production environments and to reduce the amount of power the number of instances used for VMs must be reduced. When the VMs which exhibit similar behavior characteristics are combined then amount of time required for monitoring can be reduced [18].In order to reduce the operating cost the consolidation of VMs must be performed because it helps in reducing the cost required. The VM consolidation process is automated in order to assign the servers in an adaptive fashion with the objective of power cost and network cost reduction. The problem can be studied as a M-convex based optimization which reduces the number of times the servers are switched between on/off states. The scalability of the framework increases due to its distributed nature [19].

When the host systems are over-utilized then live migration can be done on the cloud system using dynamic consolidation of VMs. The selection of VMs in an optimized way from such a overloaded host for migration is important factor. The policies are created to reduce the time duration $\mathrm{T}$ for migration, amount of duration the host is over-utilized and number of migrations required for consolidation. The policy must also take into consideration the SLA levels and also provides 'tuning parameters to achieve performance efficiency [15].

The consolidation aim is achieved with the help of Oversubscription in order to reduce cost and energy consumed. When the graph of consolidation and overload is drawn, when the consolidation value is large then cloud server will be overloaded. This overloading and Quality of Service is inversely proportional. When a set of resources co-operate with each other than overload can be reduced, live migration also helps in reduction of overload, making use of either supervised or unsupervised machine learning in a proactive scope also helps in reducing overload [16].

The cloud service providers can make use of data provided by users to improve the resource utilization. Utilizing the information in a right format to reduce the energy consumption and improve efficiency of energy is a challenging assignment. The user information is transformed; data extensive research is performed in order to dynamically make few PMs to have a sleep mode leading to between energy efficiency [17].

The main function $\mathrm{F}$ has a min (PMs) in order to achieve better energy management and load distribution. The workload in each of the PMs is computed, then partitions are created for PMs, PMs are assigned a unique group. Game based consolidation is executed to have running in a highly energy efficient manner [18].

\section{PROPOSED METHOD}

The consolidation of VM has certain design challenges which are depicted in the Fig.2. When the VM is switched between two hosts, the memory transfer must happen to the destination node and CPU transfer cannot be possible because of transition nature of VM. This throws up a challenge of number of VM migrations to keep at minimum level to meet the threshold set in the SLA policy defined by customers.

The VM consolidation can be defined as either a packaging task or a set of sub tasks which has to be taken care. The three steps play major role for VM consolidation a) Find the load values for data centers and if the load is higher a threshold $\mathrm{T}$ then migrate the VMs to a data center which is active. b) Find the VMs which needs migration and c) Find the location for VMs in either active or reactive host.

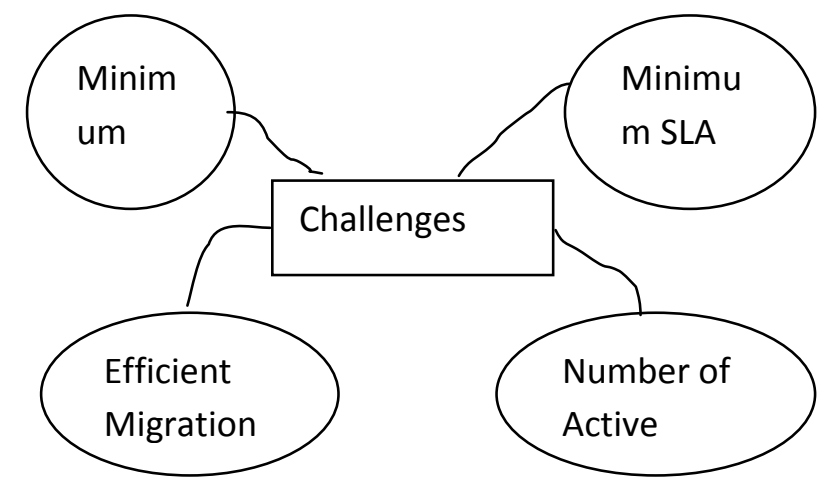

Fig.2 Challenges for VM Consolidation

Fuzzy based VM selection with controlled migration is used to achieve good efficiency in energy and delay profile. Standard Deviation, median and mean metrics are computed to identify overloading. Many data center and VMs are created to process the request generated from users and to process those requests. The migration task will change the status of host to INACTIVE to reduce the overloading scenarios. 
The VM consolidation is a multi-objective process with first process being selection of data center which has to be turned to sleep mode so that balance is established with respect to data center which is overloaded and this process will also reduce the overall energy levels. The migration process also involves a step to check the under loaded virtual machine so that overloaded data center can be migrated. At a given time instant $\mathrm{T}$, when the virtual machine will be in active stage other virtual machines will be switched to inactive state.

In the existing methods two major problems, the first one is the virtual machine which has to be used, the second one is to find out whether the load $\mathrm{L}$ on the virtual machines exceeds Th Where Th is Overload threshold. The combination of threshold and prediction based techniques is responsible for identifying the task, executing the task, compute the current capacity of virtual machine, compare the capacity $\mathrm{C}$ with a threshold number $\mathrm{TN}$, if $\mathrm{C}<=\mathrm{TN}$ then Virtual machine is under loaded otherwise virtual machine is overloaded. The existing methods have the following disadvantages are more energy consumption, power consumption and delay consumption.

To have lesser emery and delay values, fuzzy based virtual machine selection is equipped with migration process. The computation of standard deviation, mean and median based value are used to find the overload detection. The method will improve the efficiency value in the cloud. The requests are taken and then processing is performed by making use of multiple data center and virtual machines. The identification of free host in order to reduce the energy and power values, few virtual machines status is changed to inactive state if the overload is low.

The advantages of the proposed method are- reduction in energy consumption with respect to unwanted reasons, delay produced in data processing, reducing the power consumption and lower cost. The System architecture for the proposed system can be described in Fig3.

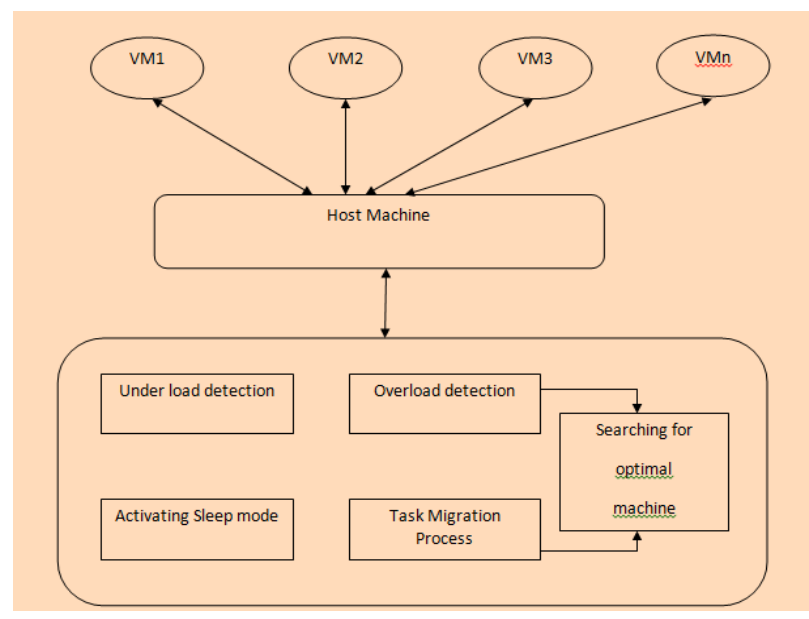

Fig.3 System Architecture

The various modules for the proposed system can be described as below

\section{Virtual machine creation}

Different kind of Virtual Machines is created based on speed, memory and bandwidth. VM is defined as a broker agent of data center which represents a user. The various activities are abstracted namely creation of VM, cloudlets submission for the VMs and destroying the VMs

\section{Virtual machine categorization}

The clustering labels for the VMs are Low Config VM, Medium Config VM and High Config VM. The mean, standard deviation and variance value are computed before assigning the cluster label for the VM.

The file size can be computed based on the following equation

$$
F S=\frac{F S \text { in } k b}{1024}
$$

Where, FS is File Size

The value of mean is computed using the following equation

$$
\mu=\frac{\sum_{i=1}^{k}(\text { speed }+ \text { memory }+ \text { bandwidth }(k i)}{3(k)}
$$

Where,

\section{$\mathrm{K}=$ an index for $\mathrm{VM}$}

The value for variance is computed using the following equation

$$
\sigma=(\mu-F S \text { inkb)*( }(\mu-F S \text { in } k b)
$$

The standard deviation can be computed using the following

$$
S D=\sqrt{\sigma}
$$

The algorithm used for VM consolidation is described in the Fig 4.

\section{Task allocation}

The data from the VM is taken as an input parameter. Based on the data available at the VMs they are assigned to host systems and finally data center is given for VM. A set of available hosts are equipped with the VM, the host is assigned a sleeping mode. During each iteration, the task allocation process is executed. 


Algorithm: VM Consolidation
Input: $N_{H}$
Process:
a) Generate an interface with respect to cloud
b) Create the VMs
c) Assign $N_{H}$ to the VMs
d) Create the Cloudlet
e) Assign the Cloudlet to VMs
f) For a given T
g) Compute the Load Detection Ratio (LDR)
h) If the Load Detection Ration is lower than a
i) If LDR>TN then VM is overloaded
j) Find the Hosts System which are overloaded
k) VM is selected from the overloaded host
1) The VM is copied to the available data center
m) The QoS value is predicted

Fig4: VM Consolidation

\section{Task Migration}

After every time duration $\mathrm{T}$, the execution of under load detection process is done, the classification of hosts is performed and then cluster of hosts which are less utilized are kept under sleep mode. This is done by making the transfer of all the VM into another active VM. During the classification process even overloaded hosts are found out and then VMs are identified from hosts in order to perform migration activity. The overloaded VMs are kept either in free hosts or a host is turned on from sleep mode.

The Fuzzy based VM selection Algorithm and Migration control is summarized in the Fig 5 .

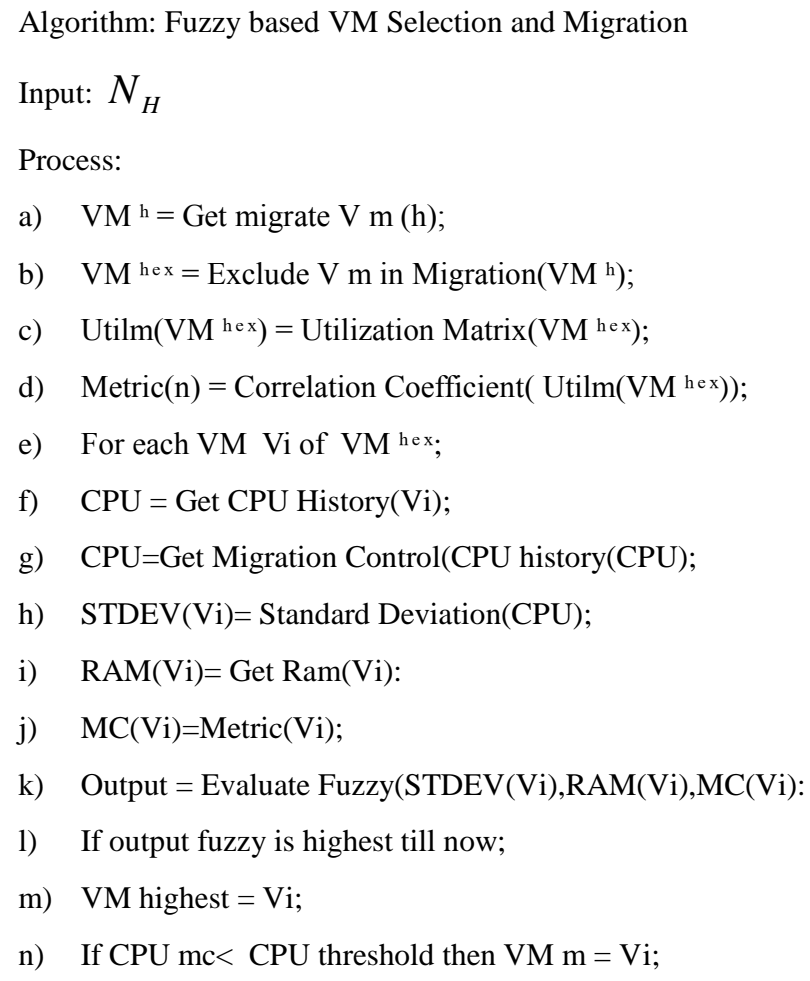

Fig 5. Fuzzy VM Selection and Migration

There are various activities that are performed in the application by the various actors namely user or an actor. The activities can be summarized in Fig 6. The user will perform the sign up once the sign up is done the user perform the login. If the authentication is successful then user is allowed to login otherwise the user is thrower out of the application. Once the user performs the login into the application the user can upload the file. The file is stored in the allocated VM and later can even perform the download of the file. The admin will perform the login into the application, once the login of the application is performed, the admin will be able to see all the registered user details, admin will be able to create a VM, admin will be able to search in the available VMs, the admin can allocated the VMs to the users based on the load and also admin will be able to view the VMs allocated to the user. 


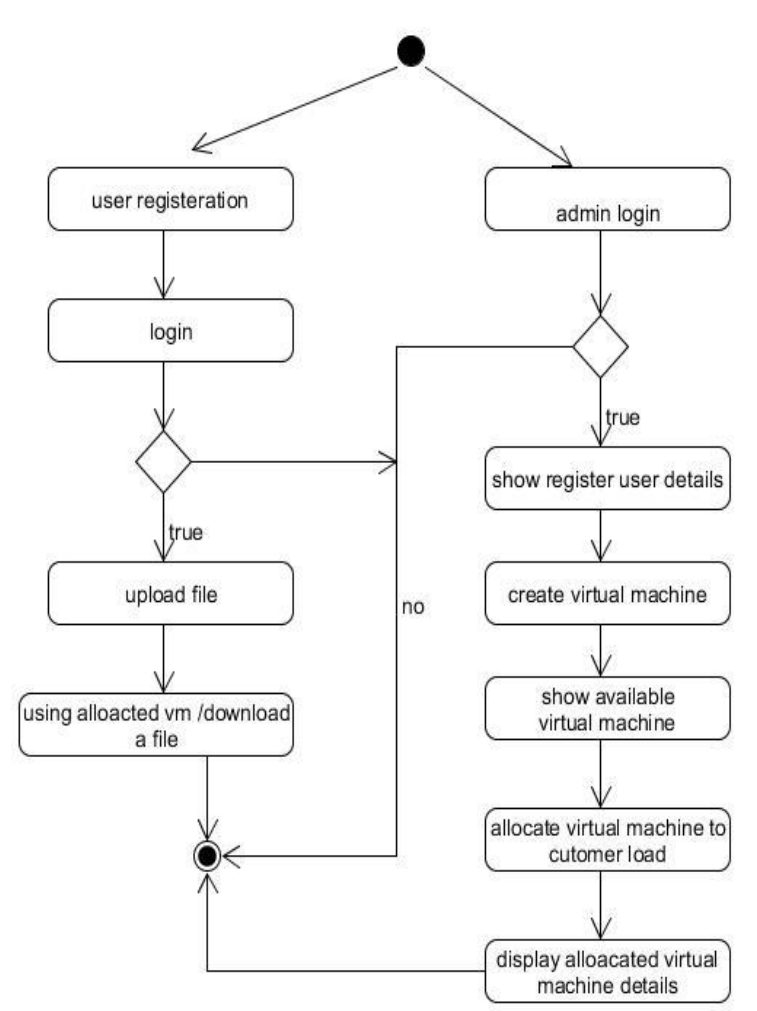

Fig 6. Activity for Proposed System

\section{RESULTS AND DISCUSSSION}

This section describes the implementation results of the project which have been developed using JAVA language.

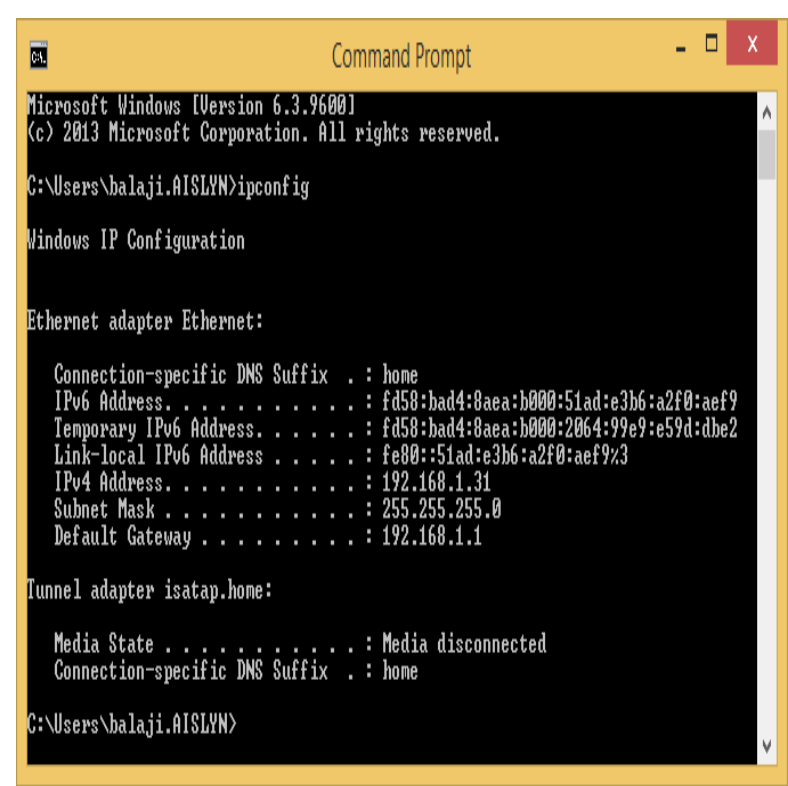

Fig7. Source System IP Address Detection

Fig 7 shows the process in which the user and the server are connected using WIFI protocol or any other adhoc connection and test is made whether the user and the server are connected or not. The IP address of the system is found out by executing the ipconfig command on the command prompt of windows system.

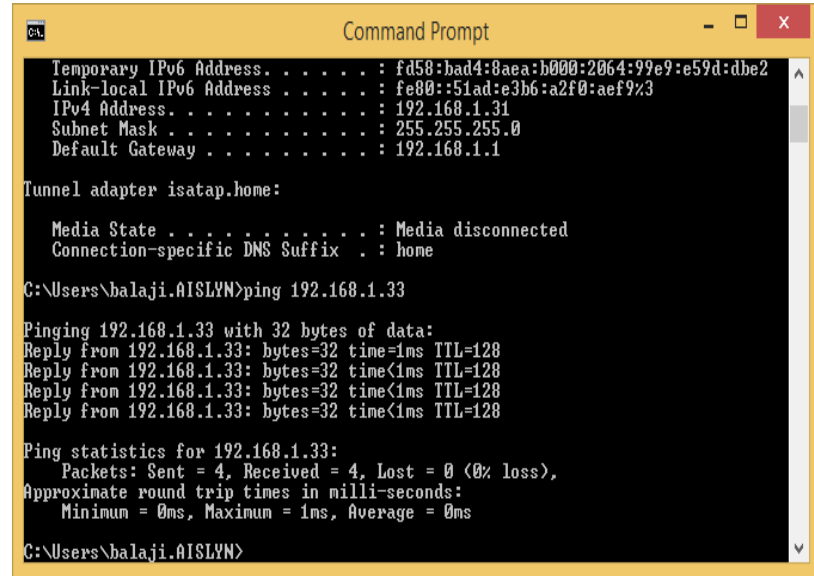

Fig 8. Communication Checking

Fig 8 shows the communication between the user and server is successful or not by executing the ping command on the server ip address. As shown in the Fig 8. The communication is successful and the loss percentage is 0 .

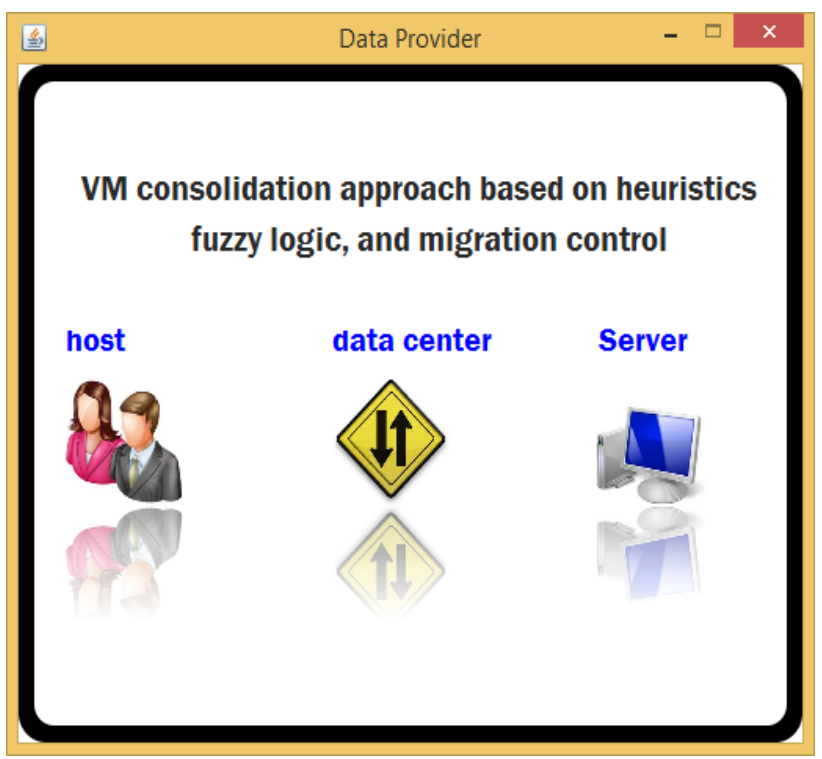

Fig 9. Home Page for Application

Fig 9 shows the various links which are available in the home screen. As shown in the screen the three major important links are host, data center and server.

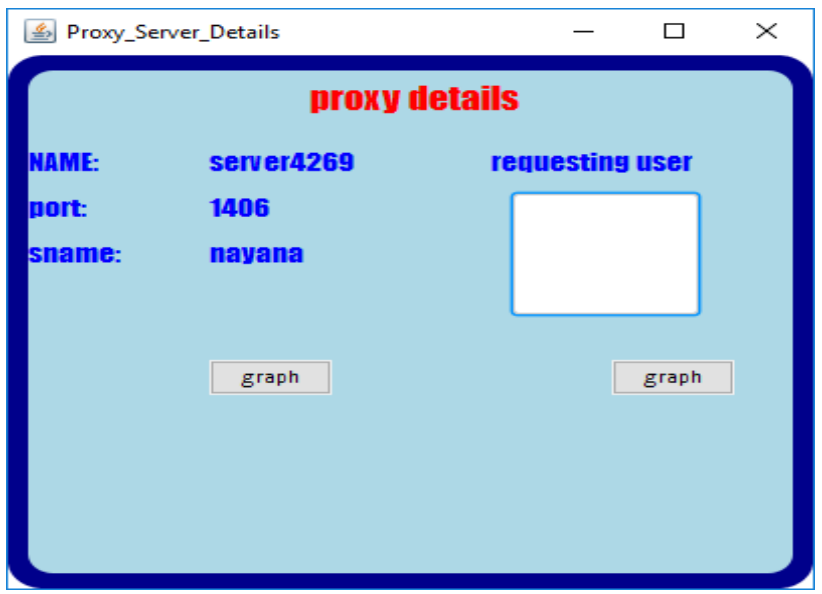

Fig 10. Proxy Server Details 
Fig 10. Shows the proxy server details. As shown in the Fig. 10 the name of the server is provided, port of the server, name of the user and the requesting user details are provided in the text area and then graph is generated.

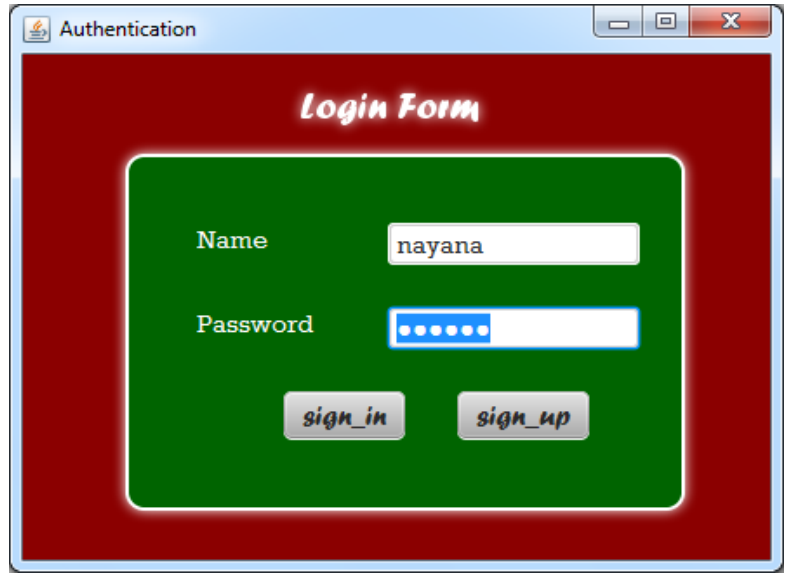

Fig 11. Login Form

Fig 11 shows the login form in which the username and password are fed for the user. If the authentication is successful the user will be able to perform various actions otherwise the authentication is failed.

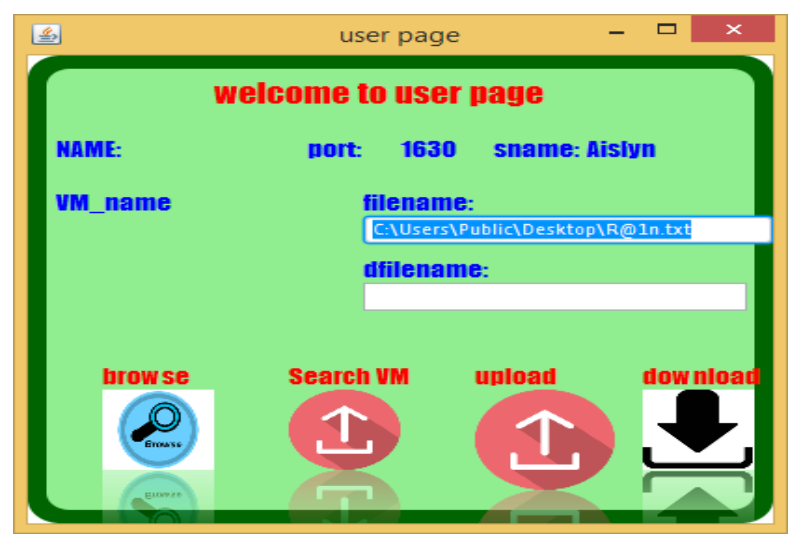

Fig 12. User Home Page

Fig 12 shows the home page after the successful login by the user. As shown in the Fig. 12 the file name is provided for the user. When the user clicks search VM icon then a request is send to the server to obtain the details.

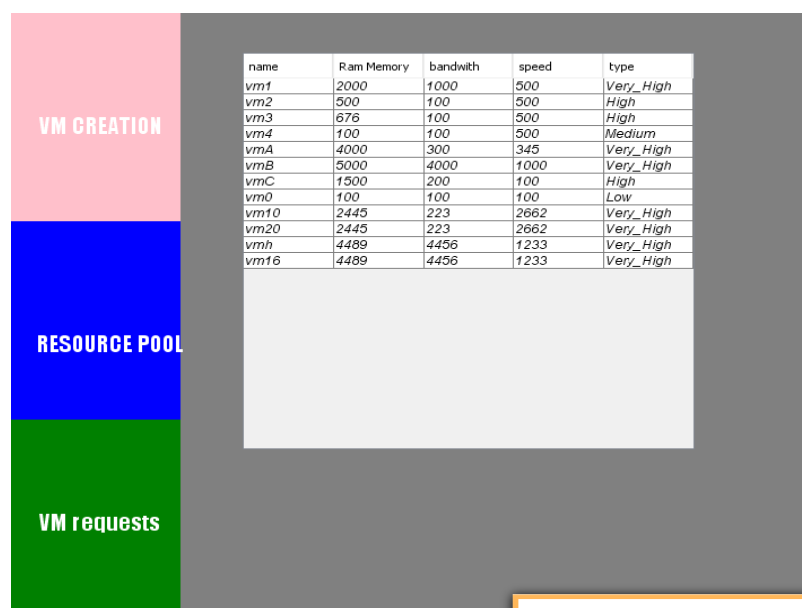

Fig 13. VM Search Details
Fig 13. Shows the VM search details, as shown in the fig there are multiple columns namely name, Ram Memory, Bandwidth, speed and type. Name column corresponds to virtual machine name, Ram Memory is the memory in GB, Bandwidth is the bandwidth value for the column, speed is the speed for the virtual machine and the final column is type column which can either be Very High, High, Medium, and Low.

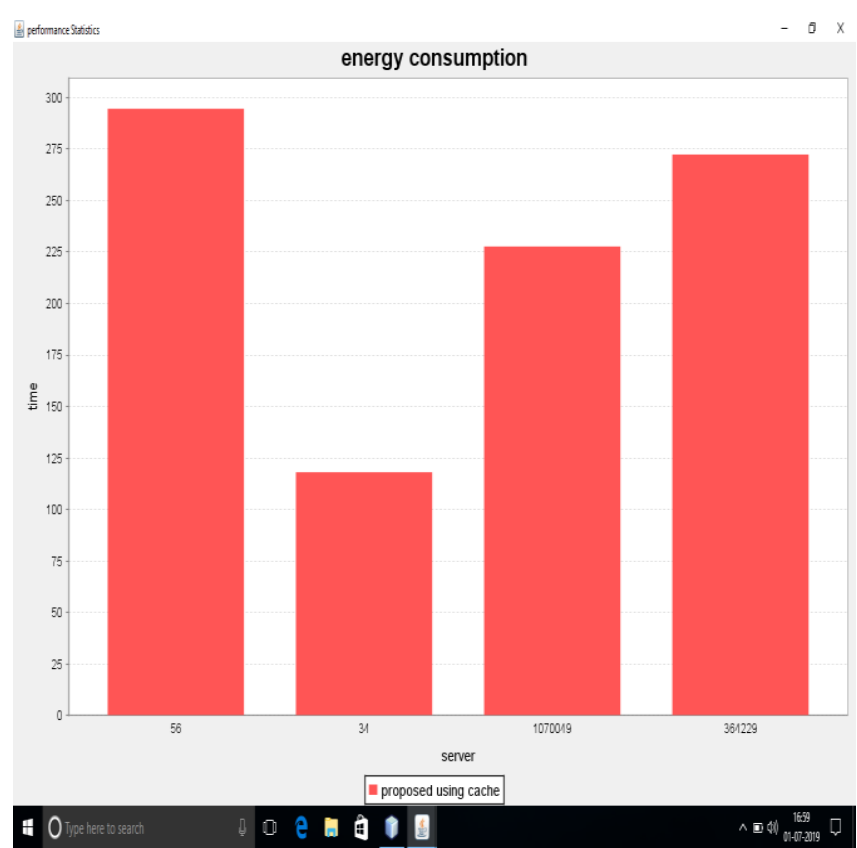

Fig 14. Energy Consumption for VM

Fig 14. Shows the energy consumption graph with respect to the virtual machines

\section{CONCLUSION}

The VM consolidation process will perform migration in an optimized way. The Fuzzy based selection process will perform an intelligent step to select the best VM which is responsible for migration from Host A to Host B, After that the system will compute the mean value computation, medium based computation, standard deviation computation for computing overload detection ratio. The proposed method has more residual energy, less delay, low cost and has less SLA violation.

\section{REFERENCES}

[1] Belady C (2011) Projecting annual new datacenter construction market size. Technical Report. Microsoft Corp., US

[2] Zhan ZH, Liu XF, Gong YJ, Zhang J, Chung HSH, Li Y (2015) Cloud computing resource scheduling and a survey of its evolutionary approaches. ACM Computing Surveys (CSUR) 47(4):63

[3] Ismaeel,Karim,Miri ,'Proactive dynamic virtual-machine consolidation for energy conservation in cloud data centers', Journal of Cloud Computing,Vol-7,2018

[4] Prevost J, Nagothu K, Jamshidi M, Kelley B (2014) Optimal calculation overhead for energy efficient cloud workload prediction. In: World Automation Congress (WAC), 2014, pp 741-747.

[5] Uddin M, Darabidarabkhani Y, Shah A, Memon J (2015) Evaluating power efficient algorithms for efficiency and 
carbon emissions in cloud data centers: A review. Renewable and Sustainable Energy Reviews 51:15531563

[6] Dabbagh M, Hamdaoui B, Guizani M, Rayes A (2015) Exploiting task elasticity and price heterogeneity for maximizing cloud computing profits.IEEE Transactions on Emerging Topics in Computing PP(99):1

[7] Fu X, Zhou C (2015) Virtual machine selection and placement for dynamic consolidation in cloud computing environment. Frontiers of Computer Science 9(2):322330

[8] Kong F, Liu X (2014) A survey on green-energy-aware power management for datacenters. ACM Computing Surveys (CSUR) 47(2):30

[9] Breitgand D, Da Silva DM, Epstein A, Glikson A, Hines MR, Ryu KD, Silva MA (2018) Dynamic virtual machine resizing in a cloud computing infrastructure. US Patent 9,858,095

[10] Gong Z, Gu X, Wilkes J (2010) Press: Predictive elastic resource scaling for cloud systems. In: 2010 International Conference on Network and Service Management, pp 916

[11] Larumbe F, Sansò B (2017) Elastic, on-line and network aware virtual machine placement within a data center. In: Integrated Network and Service Management (IM), 2017 IFIP/IEEE Symposium on. IEEE, pp 28-36

[12] Gong Z, Gu X, Wilkes J (2010) Press: Predictive elastic resource scaling for cloud systems. In: 2010 International Conference on Network and Service Management, pp 916

[13] Larumbe F, Sansò B (2017) Elastic, on-line and network aware virtual machine placement within a data center. In: Integrated Network and Service Management (IM), 2017
IFIP/IEEE Symposium on. IEEE, pp 28-36

[14] Farahnakian F, Ashraf A, Pahikkala T, Liljeberg P, Plosila J, Porres I, Tenhunen H. Using ant colony system to consolidate vms for green cloud computing

[15] Liu XF, Zhan ZH, Du KJ, Chen WN (2014) Energy aware virtual machine placement scheduling in cloud computing based on ant colony optimization approach. In: Proceedings of the 2014 conference on Genetic and evolutionary computation. ACM, pp 41-48

[16] H. Qaiser and G. Shu, "Efficient VM Selection Heuristics for Dynamic VM Consolidation in Cloud Datacenters," 2018 IEEE Intl Conf on Parallel \& Distributed Processing with Applications, Ubiquitous Computing \& Communications, Big Data \& Cloud Computing, Social Computing \& Networking, Sustainable Computing \& Communications (ISPA/IUCC/BDCloud/SocialCom/SustainCom), Melbourne, Australia, 2018, pp. 832-839.

[17] Y. Tao and S. Yu, "kFHCO: Optimal VM Consolidation via $\mathrm{k}$-Factor Horizontal Checkpoint Oversubscription," 2019 International Conference on Computing, Networking and Communications (ICNC), Honolulu, HI, USA, 2019, pp. 380-384.

[18] M. A. Khan, A. P. Paplinski, A. M. Khan, M. Murshed and R. Buyya, "Exploiting user provided information in dynamic consolidation of virtual machines to minimize energy consumption of cloud data centers," 2018 Third International Conference on Fog and Mobile Edge Computing (FMEC), Barcelona, 2018, pp. 105-114.

[19] X. Xiao, W. Zheng, Y. Xia, X. Sun, Q. Peng and Y. Guo, "A Workload-Aware VM Consolidation Method Based on Coalitional Game for Energy-Saving in Cloud," in IEEE Access, vol. 7, pp. 80421-80430, 2019. 